\title{
Vote of Thanks
}

Peer review is the life blood of this Journal. The Editors are deeply grateful to the following experts who freely gave their time during 2020 to review articles submitted for publication.

\begin{tabular}{ll} 
Andrew Argent & Busisiwe Mrara \\
Debbie Baker & Kuban Naidoo \\
Renee Blauw & Johan Nel \\
Geldine Chikanya & Vivian Norman \\
Jennifer de Beer & Peter Nourse \\
Thandiwe Dlamini & Shahed Omar \\
Reka Dulandas & Helen Perrie \\
Waheedha Emmamally & Jenna Piercy \\
Matthew Gibbs & N Plani \\
Komalan Govender & Sunita Potgieter \\
Susan Hanekom & Praksha Potgieter \\
Timothy Hardcastle & Beyra Rossouw \\
Tanya Heyns & Joan Rout \\
Lauren Hill & Shamiel Sali \\
Samantha Holzhausen & Shelley Schmollgruber \\
Jennifer Hoving & Shivani A Singh \\
Portia Janine Jordan & Juan Scribante \\
Erika Jones & David Lee Skinner \\
Nicola Kalafatis & Lincoln Solomon \\
Ismail S Kalla & Maryke Spruyt \\
Judith Kendell & Willem Stassen \\
Sharon Kling & Cindy Stephen \\
Jane Le Roux & David Thomson \\
Nkosinathi Mdladla & Heleen van Aswegen \\
Malcolm Miller & Daniël van Hoving \\
Nomaxabiso Mooi & Liezl Zuhkle \\
\hline
\end{tabular}

